\title{
Considering how biological sex impacts immune responses and COVID-19 outcomes
}

\section{Eileen P. Scully, Jenna Haverfield, Rebecca L. Ursin(D), Cara Tannenbaum and Sabra L. Klein}

Abstract $\mid$ A male bias in mortality has emerged in the COVID-19 pandemic, which is consistent with the pathogenesis of other viral infections. Biological sex differences may manifest themselves in susceptibility to infection, early pathogenesis, innate viral control, adaptive immune responses or the balance of inflammation and tissue repair in the resolution of infection. We discuss available sex-disaggregated epidemiological data from the COVID-19 pandemic, introduce sex-differential features of immunity and highlight potential sex differences underlying COVID-19 severity. We propose that sex differences in immunopathogenesis will inform mechanisms of COVID-19, identify points for therapeutic intervention and improve vaccine design and increase vaccine efficacy.

The COVID-19 pandemic, caused by the emergence of severe acute respiratory syndrome coronavirus 2 (SARS-CoV-2), has resulted in millions of infections and hundreds of thousands of deaths worldwide. Human biological sex plays a fundamental role in heterogeneous COVID-19 outcomes. Sex, defined as male, female or intersex on the basis of sex chromosome complement, reproductive tissues (ovaries or testes) and sex steroid hormone (oestrogen, progesterone and testosterone) concentrations, is a multidimensional biological characteristic that shapes infectious disease pathogenesis. We discuss how sex differences in basic molecular and cellular mechanisms can be leveraged to define the immune response to infection with SARS-CoV-2.

\section{Sex differences in COVID-19 severity The precise drivers of death, regardless of sex, in COVID-19 remain unknown. There appears to be a subset of patients in whom high levels of dysregulated inflammation lead to severe multisystem organ pathology $\mathrm{y}^{1,2}$. A postviral inflammatory syndrome has also emerged in children with COVID-19 $\left(\right.$ REFS $\left.^{3,4}\right)$. As a result, research on therapeutics has focused on both antiviral and immunomodulatory pathways ${ }^{2,5}$}

with the goal of achieving an optimized balance in immune response induction and resolution. Unfortunately, most studies fail to consider the sex of the patients, which may mask therapeutic targets.

Evidence of sex differences in COVID-19 severity emerged in China, where hospital admissions and mortality were higher among males than females ${ }^{6-8}$. In South Korea, where community testing was widespread, females represented $\sim 60 \%$ of those testing positive for SARS-CoV-2, suggesting that females acquire infection, despite having a lower case fatality rate $(\mathrm{CFR})^{9,10}$. In the United States, where testing was prioritized for people with symptomatic disease, the diagnosis rates were similar in males and females, but males had 1.5 times higher mortality ${ }^{11}$.

A male bias in COVID-19 mortality is currently reported in 37 of the 38 countries that have provided sex-disaggregated data (FIG. 1a). Our analyses show that the average male CFR across 38 countries is 1.7 times higher than the average female CFR $(P<0.0001)$ (male CFR 7.3 (95\% CI 5.4-9.2); female CFR 4.4 (95\% CI 3.4-5.5)), which is consistent with other reports ${ }^{12,13}$. There is increased risk of death for both sexes with advancing age, but at all ages above 30 years males have a significantly higher risk of death than females $(P<0.05)$ (FIG. 1 b). A male predominance of deaths from COVID-19 is consistent with what was observed in the prior SARS ${ }^{14,15}$ and Middle East respiratory syndrome (MERS) ${ }^{16}$ epidemics (caused by SARS-CoV and MERS-CoV, respectively). Although gender-related social factors, including smoking, health care-seeking behaviours and some co-morbid conditions, may impact the outcomes of COVID-19 $\left(\mathrm{REFS}^{6,17}\right.$ ) and contribute to male-female differences in disease severity, the crosscultural emergence of increased risk of death for males points to biological risk determinants. In animal models of SARS-CoV infection, differences in mortality between male and female mice were observed and were attributed to steroid hormones ${ }^{18}$. Multiple dimensions of biological sex, including sex steroids, sex chromosomes and genomic and epigenetic differences between males and females, impact immune responses ${ }^{19-26}$ and may affect responses to SARS-CoV-2 infection ${ }^{27}$.

\section{Ageing, sex and COVID-19}

Although advancing age is associated with greater risk of death in both sexes, the male bias remains evident (FIG. 1 b). An analysis of COVID-19 data from Italy, Spain, Germany, Switzerland, Belgium and Norway reveals that among all age groups older than 20 years, fatality rates are greater for males than females ${ }^{28}$. By contrast, malefemale differences in the rate of confirmed SARS-CoV-2 infections are age dependent in all countries, being greater among females aged between 10 and 50 years and greater among males before the age of 10 years and after the age of 50 years ${ }^{28}$. The age-related male-female differences in confirmed cases of SARS-CoV-2 infections are consistent with reported confirmed cases of seasonal and pandemic influenza A virus infections in Australia and Japan ${ }^{29,30}$. We interpret these data to suggest that biological sex differences contribute to male-biased death, but gender-associated risk of exposure may affect rates of infection differently for males and females.

With a focus on biology, the impact of age on susceptibility to severe COVID-19 needs to be parsed, with both immunosenescence and dysregulation of innate immune responses as potential 

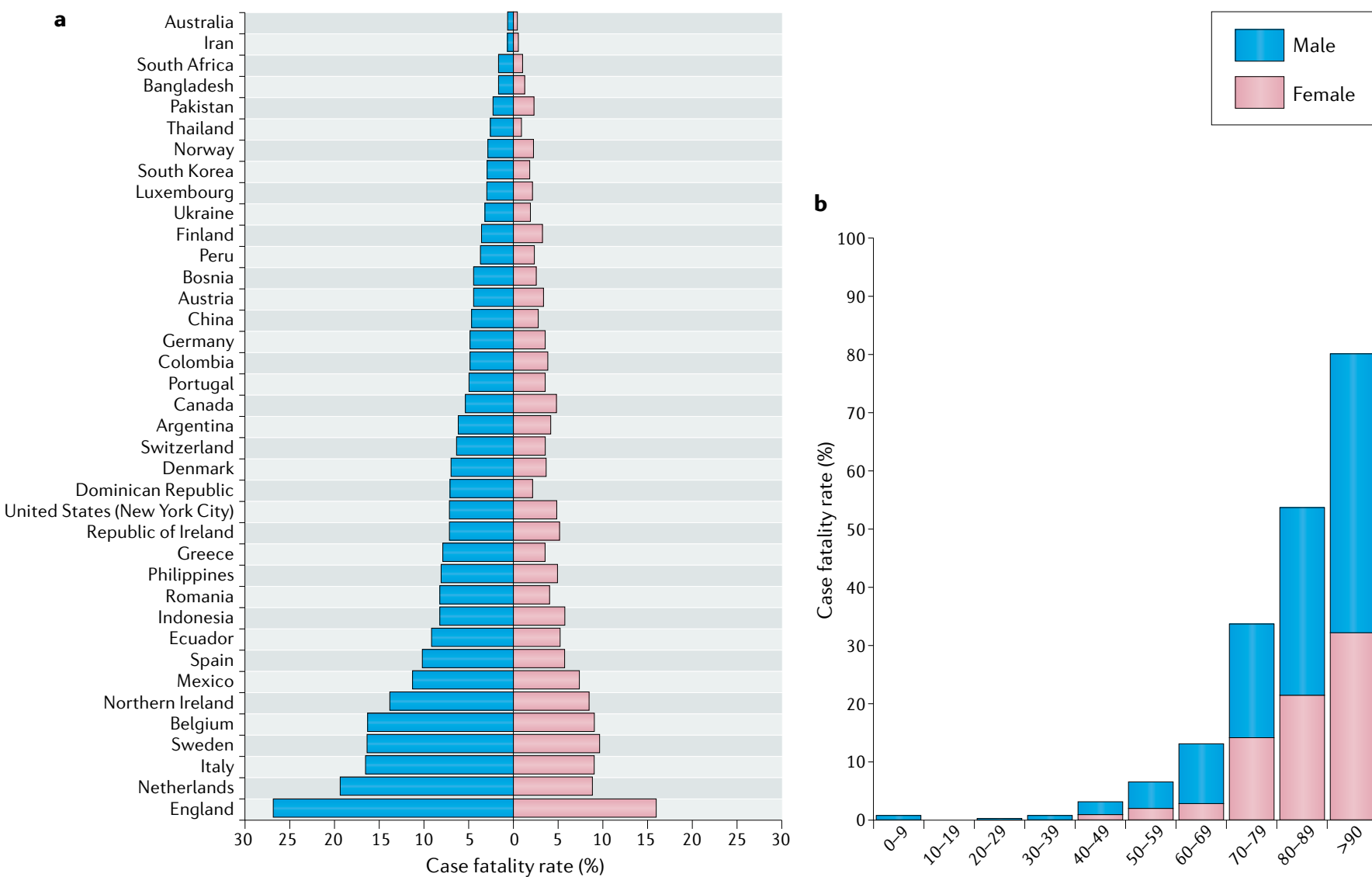

b

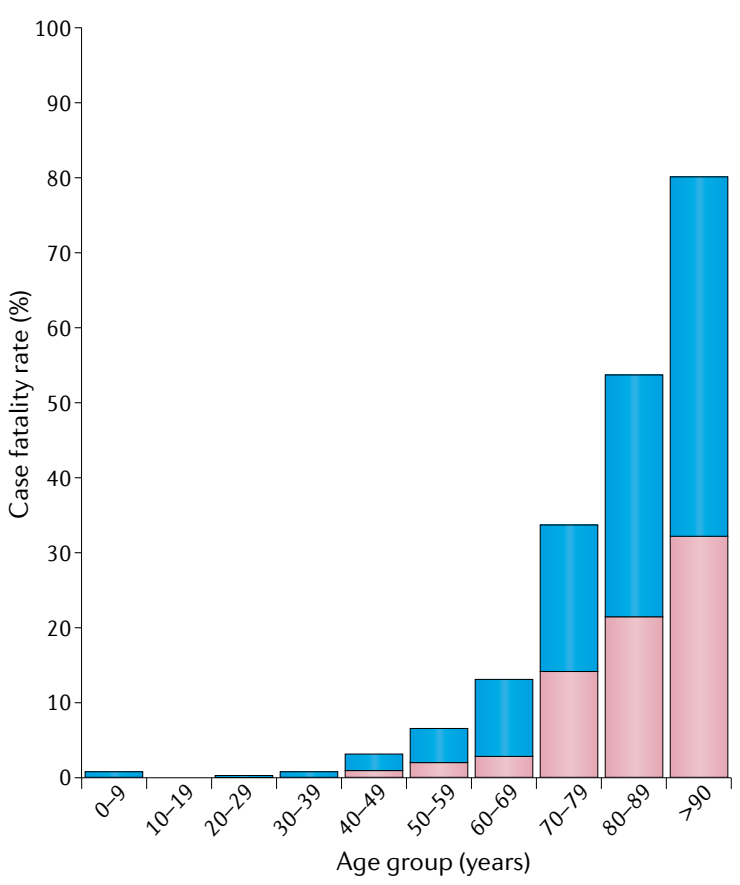

Fig. 1 | Comparative analyses of COVID-19 case fatality rates by country, sex and age. a | COVID-19 case fatality rates (CFRs) for males and females across 38 countries or regions reporting sex-disaggregated data on COVID-19 cases and deaths. CFR was calculated as the total number of deaths divided by the total number of cases for each sex multiplied by 100 . The male CFR is higher than the female CFR in 37 of the 38 regions, with an average male CFR 1.7 times greater than the average female CFR $(P<0.0001$, Wilcoxon signed rank test). $\mathbf{b} \mid$ Average COVID-19 CFRs for males and females stratified by age. The data represent 12 countries

currently reporting sex- and age-disaggregated data on COVID-19 cases and deaths (Australia, Columbia, Denmark, Italy, Mexico, Norway, Pakistan, Philippines, Portugal, Spain, Switzerland and England). The COVID-19 CFR increases for both sexes with advancing age, but males have a significantly higher CFR than females at all ages from 30 years $(P<0.05$, Wilcoxon signed rank test). The data were obtained from Global Health 50/50 and official government websites of each respective country on 7 May and 8 May 2020. For more information on the data source for a specific country, please contact the corresponding author.

mechanisms $s^{31,32}$. Biological sex differentially affects ageing of the immune system ${ }^{33}$, in part through changing concentrations of sex steroids ${ }^{34}$. In addition to reduced concentrations of sex steroids, an age-related mosaic loss of chromosome $\mathrm{Y}$ in leukocytes can alter transcriptional regulation of immunoregulatory genes ${ }^{35}$. Whether sex differences in the genomic signatures of aged immune cells translate to functional differences in the response to SARS-CoV-2 infection requires attention.

\section{Sex differences in immune responses}

Biological sex affects innate and adaptive immune responses to self and foreign antigens, resulting in sex differences in autoimmunity as well as in responses to infections and vaccines ${ }^{36,37}$. Immune cell subsets have sex-specific patterns of gene expression, with most differentially expressed genes found on autosomes, demonstrating sex-specific regulation of shared genetic material ${ }^{26}$. The sex chromosomes also directly contribute. Males are at higher risk of diseases caused by deleterious X-linked alleles. Incomplete inactivation of immunoregulatory genes on the $\mathrm{X}$ chromosome can also occur in females, which results in a gene dosage imbalance between sexes ${ }^{38,39}$. Incomplete $\mathrm{X}$ chromosome inactivation has been implicated in female-biased autoimmune diseases ${ }^{40}$ and in vaccine efficacy ${ }^{41}$. The Y chromosome has immunoregulatory function, broadly impacting immune transcriptional profiles linked to autoimmune disease $\mathrm{e}^{42}$ and impacting outcomes of influenza virus and coxsackie virus infection in animals ${ }^{43,44}$. Sex-specific features of epigenomic organization also dictate differential availability of transcriptional targets ${ }^{21,45}$. Superimposed on these genomic elements is the direct effect of sex steroid exposure. Oestrogens ${ }^{46,47}$, progesterone $^{48-52}$ and testosterone $\mathrm{e}^{53}$ have direct effects on immune cell function that are driven by the signalling of these hormones through their respective cellular receptors. The variation in sex steroid concentrations that occurs over the life course contributes to differences in immune profiles and disease susceptibility patterns at different ages ${ }^{20,52}$. Consistent with this variation, both sex and age contribute to unique transcriptional signatures of immune cells both at the baseline and after exposure to immunostimulants ${ }^{19,21,22}$. The summative effect is a sex-specific transcriptional regulatory network of genetic variants, epigenetic modifications, transcription factors and sex steroids that leads to a functional difference 
in the immune response ${ }^{25,54}$. FIGURE 2 highlights intersections between SARS-CoV-2 infection and sources of sex bias in pathophysiology that warrant further investigation.

\section{Sex bias in SARS-CoV-2 infection} Virus entry receptors. SARS-CoV-2 uses angiotensin-converting enzyme 2 (ACE2) as an entry receptor, with virus entry enhanced by cellular transmembrane serine protease 2 (TMPRSS2), which primes the spike protein of the virus ${ }^{55,56}$. ACE2 is an $\mathrm{X}$ chromosome-encoded gene that is downregulated by oestrogen ${ }^{57}$ and exhibits tissue-specific expression patterns ${ }^{39}$. Differences in ACE2 expression may be driven by sex-differential expression of $A C E 2$ variants ${ }^{58-60}$. ACE2 is associated with interferon gene expression ${ }^{61,62}$, which in turn shows sex-specific regulation. The cellintrinsic regulation of ACE2 expression may change with age, in response to changing levels of sex steroids, or following viral challenge. TMPRSS2 is regulated by androgen receptor signalling in prostate cells ${ }^{63}$. Initial investigations have not demonstrated a significant difference in TMPRSS2 mRNA expression in lung tissue from males and females, but it is unknown whether androgens may impact expression in the setting of infection with SARS-CoV-2 (REFS ${ }^{63,64}$ ) or whether the level of expression has an impact on SARS-CoV-2 burden. Further research is needed to determine whether sex-biased expression of $A C E 2$, coupled with the regulation of TMPRSS2 by androgens, increases SARS-CoV-2 susceptibility of males compared with females.

Interferons. Innate sensing of viruses, production of interferons and activation of the inflammasome are the first line of defence against viruses ${ }^{65}$. In the case of SARS-CoV-2, where there is no pre-existing adaptive immune memory, the success of this early antiviral response may be a determinant of disease outcome. Innate sensing of viral RNA by the patternrecognition receptor Toll-like receptor 7 (TLR7) is sex biased, as TLR7 escapes $\mathrm{X}$ chromosome inactivation, resulting in greater expression in female immune cells; this has also been linked to sex differences in autoimmunity ${ }^{40,66}$ and vaccine efficacy ${ }^{41}$. There is greater production of interferon- $\alpha$ (IFN $\alpha$ ) from plasmacytoid dendritic cells from adult females than from adult males ${ }^{67,68}$, an effect modulated by sex steroids ${ }^{69-71}$. In animal models of SARS-CoV infection, pretreatment with pegylated IFNa was associated with protection of lung tissue ${ }^{72}$ but without consideration of biological sex. In SARS$\mathrm{CoV}-2$, emerging data suggest that there is aberrant activation of interferon responses but preserved chemokine signalling, which has been postulated to contribute to immunopathology ${ }^{73}$. Studies are needed

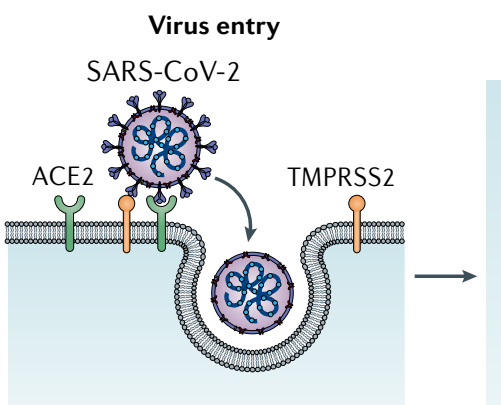

ACE2

- X-linked

- Downregulated by oestrogens

- Sex differential impact of

ACE2 variants in hypertension

TMPRSS2

- Androgen regulated in prostate cells
Virus sensing/ early antiviral response

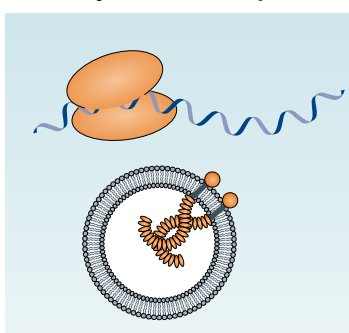

- TLR7 X-linked with biallelic expression in females

- Increased IFNa from pDCs in response to TLR7 stimulation

pDC responses are sex hormone regulated
Innate immune response

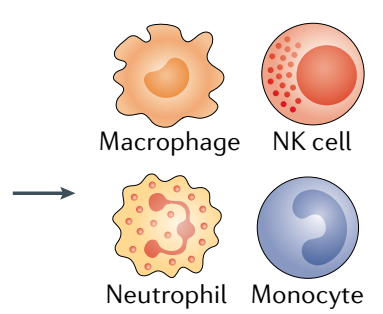

- Sex differences in cytokine and chemokine production and neutrophil recruitment in influenza

- Sex differential gene expression in innate cell subsets
Adaptive immune response
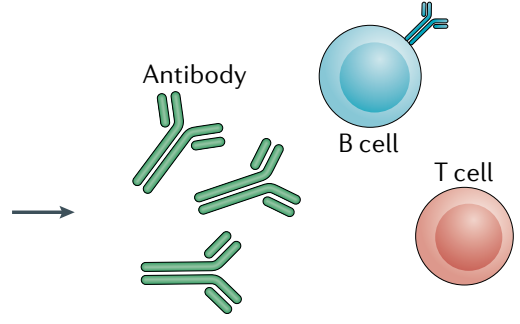

$\downarrow$ B cell activity and adaptive immunity with age in males

Antibody response to $\mathrm{H} 1 \mathrm{~N} 1$ vaccine in male mice

$\uparrow$ Somatic hypermutation, germinal centre formation and epigenetic accessibility of B cell loci in females

T cell subset distribution and activation affected by oestrogens

- Sex-specific development of regulatory $T$ cells

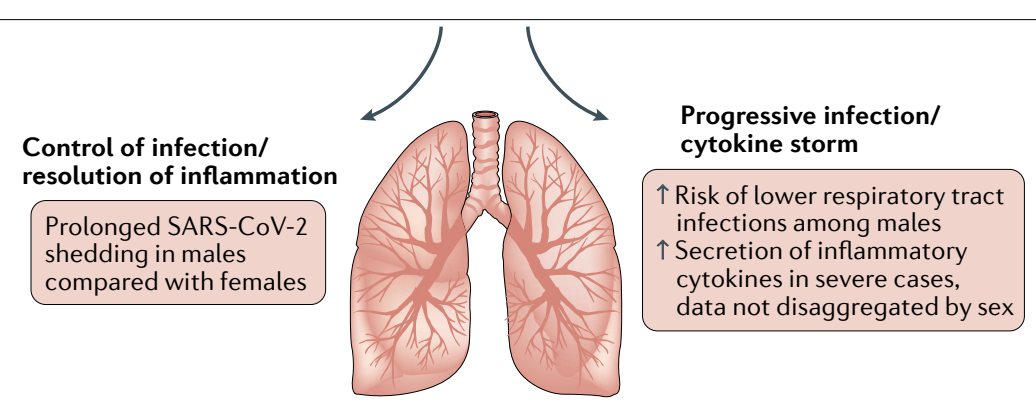

Fig. 2 | Known sex differences that may impact immune responses to SARS-CoV-2 and COVID-19 progression. An illustrative summary of the sequence of events in severe acute respiratory syndrome coronavirus 2 (SARS-CoV-2) infection and the associated immune responses. Broadly speaking (from left to right), there are the initial steps of virus entry, innate recognition of the virus with activation of antiviral programmes, the recruitment of innate immune cells and induction of an adaptive immune response.
These major steps culminate either in successful control of infection and pathogen elimination or in a pathological inflammatory state. Sex differences that may be operative at multiple points along these pathways are indicated in the blue boxes. ACE2, angiotensin-converting enzyme 2; H1N1, H1N1 influenza virus; IFNa, interferon- $\alpha$; NK, natural killer; pDC, plasmacytoid dendritic cell; TLR7, Toll-like receptor 7; TMPRSS2, transmembrane protease serine 2 . 
to determine whether differences in the magnitude or kinetics of the interferon response may contribute to a sex bias in the early control or severity of SARS-CoV-2 infection and may inform considerations of interferons as therapies for COVID-19 $\left(\mathrm{REF}^{74}\right)$. Early data suggest that male sex may be associated with a longer duration of viral detection, even within families ${ }^{75,76}$, raising the question of whether females have more efficient clearance of the virus. The rate of virus clearance will need to be assessed in evaluating the efficacy of innate and adaptive immune responses.

\section{Adaptive immunity. Females generally} mount greater antibody responses to viral infection and vaccination, albeit with higher levels of autoreactivity ${ }^{77}$. The mechanisms for sex differences in antibody production include oestrogenic enhancement of somatic hypermutation $^{78}$, less stringent selection against autoreactive B cells $\mathrm{s}^{77,79-82}$ and sex differences in germinal centre formation ${ }^{83}$ and in the epigenetic accessibility of B cell $\operatorname{loci}^{21}$. It is still unknown whether sex has an impact on antibody generation in SARS$\mathrm{CoV}-2$ infection. Early studies suggest that titres of antibodies to some viral epitopes are higher in patients with severe COVID-19 and that seroconversion may not be tightly linked to declining virus titres ${ }^{84,85}$. Ongoing studies evaluating the infusion of convalescent serum may provide answers as to the protective capacity of these antibodies $^{86}$, but these studies are currently not considering biological sex. Generation of protective, neutralizing antibodies is a goal of vaccine development, with the cautionary note that in models of SARS-CoV vaccination some antibody responses induced potent inflammatory responses ${ }^{57}$. Persistence of antibodies, epitope targeting and non-neutralizing Fc-mediated antibody characteristics should be assessed with sex-stratified analyses. As vaccines are developed, the female bias towards both potent responses and adverse effects should be considered and sex-specific dosing should be tested, where appropriate ${ }^{87}$.

Sex impacts the development of regulatory $\mathrm{T}$ cells ${ }^{88-91}$, the distribution of lymphocyte subsets ${ }^{92}$ and the overall quality of T cell responses ${ }^{93,94}$. In T cells, overexpression of X-encoded immune genes, including CD40LG and CXCR3, has been linked to incomplete $\mathrm{X}$ chromosome inactivation and $\mathrm{T}$ cell-specific epigenetic modifications of the X chromosome ${ }^{95,96}$. It is unknown whether $\mathrm{T}$ cell phenotypes contribute to COVID-19; data from the prior SARS outbreak did not link T cell responses to outcomes in humans ${ }^{97}$, but mouse models suggest a role for $\mathrm{CD} 4^{+}$ $\mathrm{T}_{\text {cells }}{ }^{98}$. In patients with MERS, T cell responses were dysregulated ${ }^{99}$, but sex differences were not analysed. In the current pandemic, lymphopenia is associated with severe disease ${ }^{100,101}$, and early evidence suggests that the clinical markers of lymphocyte count may be lower in males and neutrophil-lymphocyte ratios may be higher ${ }^{17}$. Further work is needed to define the sex-differential role of $\mathrm{T}$ cells in acute infection, in acute lung injury phenotypes ${ }^{102}$ and as potential vaccine targets.

\section{Severe infection and acute respiratory distress syndrome. Severe cases of} COVID-19 are typically marked by acute respiratory distress syndrome (ARDS), with respiratory failure requiring oxygen support and mechanical ventilation. The infection is primarily characterized by diffuse alveolar damage without a consistent pattern of cell infiltration ${ }^{75,103-105}$. The pathogenesis of ARDS involves the disruption of normal barrier function, inflammation and subsequent tissue repair. Whether there are sex-specific risks for ARDS and death from other causes, such as trauma, remains unknown ${ }^{106,107}$, although there is a suggestion of a higher risk of lower respiratory tract infections among males ${ }^{108}$ and that steroid hormones modulate the immune response to respiratory viral pathogens ${ }^{109}$. In one cohort of patients with COVID-19, severe respiratory failure was associated with a pattern of inflammation, macrophage activation and depletion of lymphocytes that was distinct from bacterial infection $^{110}$. There was a sex bias for severe COVID-19 not observed in the comparator group with bacterial infections ${ }^{110}$. Sexdifferential production of IL-6 (REF. ${ }^{111}$ ), monocyte transcriptional patterns and inflammatory set point ${ }^{19,21,22}$ could contribute to an enhanced risk of death in males and highlight the importance of sex-stratified analyses to guide deployment of safe and effective immunomodulatory therapeutics for males and females ${ }^{112}$.

\section{Conclusions}

Emerging data demonstrating more favourable outcomes for communitydwelling adult females across age strata offer an immediate opportunity for comparative biology experiments to define features of COVID-19 pathogenesis and the associated immune response. The research pipeline should integrate sex as a biological variable in all stages, from fundamental research to preclinical drug development, clinical trials and epidemiological analyses ${ }^{113}$. Considering the role of intersecting factors - including, but not limited to, gender, age, race and other identifying characteristics - is critical to understanding the biological and sociocultural factors contributing to heterogeneous COVID-19 outcomes. Sex is a driver of discovery and innovation ${ }^{114}$, and taking a sex-informed approach to COVID-19 research ${ }^{115}$ and medicine ${ }^{116}$ will uncover novel features of the host immune response to SARS-CoV-2 and ultimately result in more equitable health outcomes.

Eileen P. Scully', Jenna Haverfield ${ }^{2}$, Rebecca L. Ursin D $^{3}$ Cara Tannenbaum ${ }^{2,4}$ and Sabra L. Klein ${ }^{3,5 凶}$

'Division of Infectious Diseases, Department of Medicine, Johns Hopkins University, Baltimore, $M D, U S A$.

${ }^{2}$ Institute of Gender and Health, Canadian Institutes of Health Research, Montréal, Québec, Canada.

${ }^{3}$ Department of Biochemistry and Molecular Biology, The Johns Hopkins Bloomberg School of Public Health, Baltimore, MD, USA.

${ }^{4}$ Centre de Recherche de I'Institut Universitaire de Gériatrie de Montréal, Faculté de Médecine, Université de Montréal, Montréal, Québec, Canada.

${ }^{5}$ W. Harry Feinstone Department of Molecular Microbiology and Immunology, The Johns Hopkins Bloomberg School of Public Health, Baltimore, $M D, U S A$.

凶e-mail: sklein2@jhu.edu

https://doi.org/10.1038/s41577-020-0348-8

Published online 11 June 2020

1. Mehta, P. et al. COVID-19: consider cytokine storm syndromes and immunosuppression. Lancet 395, 1033-1034 (2020).

2. Berlin, D. A., Gulick, R. M. \& Martinez, F. J. Severe Covid-19. N. Engl. J. Med. https://doi.org/10.1056/ NEJMcp2009575 (2020).

3. Verdoni, L. et al. An outbreak of severe Kawasaki-like disease at the Italian epicentre of the SARS-CoV-2 epidemic: an observational cohort study. Lancet 395 , 1771-1778 (2020).

4. Centers for Disease Control and Prevention. Multisystem inflammatory syndrome in children (MIS-C) associated with coronavirus disease 2019 (COVID-19). CDC https://emergency.cdc.gov/ han/2020/han00432.asp (2020)

5. Vabret, N. et al. Immunology of COVID-19: current state of the science. Immunity https://doi.org/ 10.1016/j.immuni.2020.05.002 (2020).

6. Chen, N. et al. Epidemiological and clinical characteristics of 99 cases of 2019 novel coronavirus pneumonia in Wuhan, China: a descriptive study. Lancet 395, 507-513 (2020).

7. Guan, W. J. et al. Clinical characteristics of coronavirus disease 2019 in China. N. Engl. J. Med. 382 1708-1720 (2020).

8. Wu, Z. \& McGoogan, J. M. Characteristics of and important lessons from the coronavirus disease 2019 (COVID-19) outbreak in China: summary of a report of 72314 cases from the Chinese center for disease control and prevention. JAMA https://doi.org/10.1001/ jama.2020.2648 (2020).

9. Dudley, J. P. \& Lee, N. T. Disparities in age-specific morbidity and mortality from SARS-CoV-2 in China and the Republic of Korea. Clin. Infect. Dis. https:// doi.org/10.1093/cid/ciaa354 (2020).

10. Ministry of Health and Welfare of South Korea. Domestic occurrence status. MOHW http: ncov.mohw.go.kr/bdBoardList_Real.do?brdld= 1 \&brdGubun= 11 \&ncvContSeq=£contSeq=\&board d $=$ ¿gubun $=$ (2020).

11. NYC. COVID-19: data. NYC.gov https://www1 nyc.gov/ site/doh/covid/covid-19-data.page (2020). 
12. Jin, J.-M. et al. Gender differences in patients with COVID-19: focus on severity and mortality. Front. Public Health https://doi.org/10.3389/ fpubh.2020.00152 (2020)

13. Peckham, H. et al. Sex-bias in COVID-19: a metaanalysis and review of sex differences in disease and immunity. Preprint at ResearchSquare https://doi.org 10.21203/rs.3.rs-23651/v2 (2020).

14. Karlberg, J., Chong, D. S. \& Lai, W. Y. Do men have a higher case fatality rate of severe acute respiratory syndrome than women do? Am. J. Epidemiol. 159, 229-231 (2004).

15. Leong, H. N. et al. SARS in Singapore-predictors of disease severity. Ann. Acad. Med. Singap. 35, 326-331 (2006)

16. Alghamdi, I. G. et al. The pattern of Middle East respiratory syndrome coronavirus in Saudi Arabia: a descriptive epidemiological analysis of data from the Saudi Ministry of Health. Int. J. Gen. Med. 7 , 417-423 (2014).

17. Meng, Y. et al. Sex-specific clinical characteristics and prognosis of coronavirus disease-19 infection in Wuhan, China: a retrospective study of 168 severe patients. PLOS Pathog. 16, e1008520 (2020).

18. Channappanavar, R. et al. Sex-based differences in susceptibility to severe acute respiratory syndrome coronavirus infection. J. Immunol. 198, 4046-53. (2017).

19. Bongen, E. et al. Sex differences in the blood transcriptome identify robust changes in immune cell proportions with aging and influenza infection. Cell Rep. 29, 1961-73 e4 (2019).

20. Ghosh, S. \& Klein, R. S. Sex drives dimorphic immune responses to viral infections. J. Immunol. 198 1782-90. (2017)

21. Marquez, E. J. et al. Sexual-dimorphism in human immune system aging. Nat. Commun. 11, 751 (2020)

22. Piasecka, B. et al. Distinctive roles of age, sex, and genetics in shaping transcriptional variation of human immune responses to microbial challenges. Proc. Nat Acad. Sci. USA 115, E488-E497. (2018).

23. Schurz, $\mathrm{H}$. et al. The $\mathrm{X}$ chromosome and sex-specific effects in infectious disease susceptibility. Hum. Genomics 13, 2 (2019).

24. vom Steeg, L. G. \& Klein, S. L. SeXX matters in infectious disease pathogenesis. PLoS Pathog. 12, e1005374 (2016).

25. Ober, C., Loisel, D. A. \& Gilad, Y. Sex-specific genetic architecture of human disease. Nat. Rev. Genet. 9 911-922 (2008)

26. Schmiedel, B. J. et al. Impact of genetic polymorphisms on human immune cell gene expression. Cell 175, 1701-15.e16 (2018).

27. Klein, S. L. et al. Biological sex impacts COVID-19 outcomes in the United States. PLoS Pathog. (in the press)

28. Marina S. \& Piemonti L. Gender and age effects on the rates of infection and deaths in individuals with confirmed SARS-CoV-2 infection in six European countries. Lancet https://doi.org/10.2139/ ssrn.3576790 (2020).

29. Wong, K. C., Luscombe, G. M. \& Hawke, C. Influenza infections in Australia 2009-2015: is there a combined effect of age and sex on susceptibility to virus subtypes? BMC Infect. Dis. 19, 42 (2019)

30. Eshima, N. et al. Sex- and age-related differences in morbidity rates of 2009 pandemic influenza A H1N virus of swine origin in Japan. PLOS ONE 6, e19409 (2011)

31. Koff, W. C. \& Williams, M. A. Covid-19 and immunity in aging populations - a new research agenda. N. Engl J. Med https://doi.org/10.1056/ NEJMp2006761 (2020).

32. Nikolich-Zugich, J. et al. SARS-COV-2 and COVID-19 in older adults: what we may expect regarding pathogenesis, immune responses, and outcomes. GeroScience 42, 505-514 (2020).

33. Leng, S. X. \& Margolick, J. B. Aging, sex inflammation, frailty, and CMV and HIV infections. Cell Immunol. 348, 104024 (2020).

34. Potluri, T. et al. Age-associated changes in the impact of sex steroids on influenza vaccine responses in males and females. NPJ Vaccines 4, 29 (2019).

35. Dumanski, J. P. et al. Immune cells lacking $Y$ chromosome have widespread dysregulation of autosomal genes. Preprint at bioRxiv https://doi.org/ 10.1101/673459 (2020).

36. Klein, S. L. \& Flanagan, K. L. Sex differences in immune responses. Nat. Rev. Immunol. 16, 626-638 (2016)

37. Markle, J. G. \& Fish, E. N. SeXX matters in immunity. Trends Immunol. 35, 97-104 (2014).
38. Carrel, L. \& Brown, C. J. When the Lyon(ized chromosome) roars: ongoing expression from an inactive X chromosome. Philos. Trans. R. Soc. Lond. B Biol. Sci. 372, 20160355 (2017)

39. Tukiainen, $\mathrm{T}$. et al. Landscape of $\mathrm{X}$ chromosome inactivation across human tissues. Nature $\mathbf{5 5 0}$ 244-248 (2017).

40. Souyris, M. et al. TLR7 escapes X chromosome inactivation in immune cells. Sci. Immunol. 3 eaap8855 (2018).

41. Fink, A. L., Engle, K., Ursin, R. L., Tang, W. Y. $\&$ Klein, S. L. Biological sex affects vaccine efficacy and protection against influenza in mice. Proc. Natl Acad. Sci. USA 115, 12477-82. (2018).

42. Case, L. K. et al. The $\mathrm{Y}$ chromosome as a regulatory element shaping immune cell transcriptomes and susceptibility to autoimmune disease. Genome Res. 23, 1474-1485 (2013).

43. Krementsov, D. N. et al. Genetic variation in chromosome $\mathrm{Y}$ regulates susceptibility to influenza $\mathrm{A}$ virus infection. Proc. Natl Acad. Sci. USA 114, 3491-3496 (2017).

44. Robinson, D. P. et al. Sex chromosome complement contributes to sex differences in Coxsackievirus B3 but not influenza A virus pathogenesis. Biol. Sex. Differ. 2, 8 (2011)

45. Golden, L. C et al. Parent-of-origin differences in DNA methylation of $X$ chromosome genes in T lymphocytes. Proc. Natl Acad. Sci. USA 116, 26779-26787 (2019).

46. Straub, R. H. The complex role of estrogens in inflammation. Endocr. Rev. 28, 521-574 (2007)

47. Peretz, J., Pekosz, A., Lane, A. P \& Klein S L Estrogenic compounds reduce influenza A virus replication in primary human nasal epithelial cells derived from female, but not male, donors. Am. J. Physiol. Lung Cell Mol. Physiol 310, L415-L425 (2016).

48. Hall, O. J. \& Klein, S. L. Progesterone-based compounds affect immune responses and susceptibility to infections at diverse mucosal sites. Mucosal Immunol. 10, 1097-107. (2017).

49. Hall, O. J. et al. Progesterone-based therapy protects against influenza by promoting lung repair and recovery in females. PLoS Pathog. 12, e 1005840 (2016).

50. Hall, O. J. et al. Progesterone-based contraceptives reduce adaptive immune responses and protection against sequential influenza A virus infections. J. Virol. 91, e02160-16 (2017).

51. Vom Steeg, L. G. \& Klein, S. L. Sex steroids mediate bidirectional interactions between hosts and microbes. Horm. Behav. 88, 45-51 (2017).

52. Vom Steeg, L. G. \& Klein, S. L. Sex and sex steroids impact influenza pathogenesis across the life course. Semin. Immunopathol. 41, 189-94. (2019).

53. Furman, D et al Systems analysis of sex differences reveals an immunosuppressive role for testosterone in the response to influenza vaccination. Proc. Natl Acad. Sci. USA 111, 869-874 (2014).

54. Ellegren, H. \& Parsch, J. The evolution of sex-biased genes and sex-biased gene expression. Nat. Rev. Genet. 8, 689-698 (2007).

55. Hoffmann, M. et al. SARS-CoV-2 cell entry depends on ACE2 and TMPRSS2 and is blocked by a clinically proven protease inhibitor. Cell 181, 271-280 (2020)

56. Matsuyama, S. et al. Enhanced isolation of SARS-CoV- 2 by TMPRSS2-expressing cells. Proc Nat Acad. Sci. USA 117, 7001-7003 (2020)

57. Liu, J. et al. Sex differences in renal angiotensin converting enzyme 2 (ACE2) activity are 17 betaoestradiol-dependent and sex chromosomeindependent. Biol Sex. Differ 1, 6 (2010).

58. Zhang, Q. et al. Association of angiotensin-converting enzyme 2 gene polymorphism and enzymatic activity with essential hypertension in different gender: a case-control study. Medicine 97, e 12917 (2018).

59. Asselta, R., Paraboschi, E. M., Mantovani, A ¿ Duga, S. ACE2 and TMPRSS2 variants and expression as candidates to sex and country differences in COVID-19 severity in Italy. Preprint at medRxiv https://doi.org/10.1101/ 2020.03.30.20047878 (2020).

60. Gibson, W. T., Evans, D. M., An, J. \& Jones, S. J. ACE 2 coding variants: a potential $x$-linked risk factor for COVID-19 disease. Preprint at bioRxiv https:// doi.org/10.1101/2020.04.05.026633 (2020).

61. Ziegler, C. G. K. et al. SARS-CoV-2 receptor ACE2 is an interferon-stimulated gene in human airway epithelial cells and is enriched in specific cell subsets across tissues. Cell 181, 1016-1035 (2020).
62. Sungnak, W. et al. SARS-CoV-2 entry factors are highly expressed in nasal epithelial cells together with innate immune genes. Nat. Med. 26, 681-687 (2020).

63. Stopsack, K. H., Mucci, L. A., Antonarakis, E. S Nelson, P. S. \& Kantoff, P. W. TMPRSS2 and COVID-19: serendipity or opportunity for intervention? Cancer Discov. https://doi.org/10.1158/2159-8290.CD-20 0451 (2020).

64. Baratchian, M. et al. No evidence that androgen regulation of pulmonary TMPRSS2 explains sexdiscordant COVID-19 outcomes. Preprint at bioRxiv https://doi.org/10.1101/2020.04.21.051201 (2020).

65. Iwasaki, A. A virological view of innate immune recognition. Annu. Rev. Microbiol. 66, 177-196 (2012).

66. Souyris, M., Mejia, J. E., Chaumeil, J. \& Guery, J. C. Female predisposition to TLR7-driven autoimmunity: gene dosage and the escape from X chromosome inactivation. Semin. Immunopathol. 41, 153-64. (2019).

67. Berghofer, B. et al. TLR7 ligands induce higher IFN-alpha production in females. J. Immunol. 177, 2088-2096 (2006).

68. Meier, A. et al. Sex differences in the Toll-like receptor-mediated response of plasmacytoid dendritic cells to HIV-1. Nat. Med. 15, 955-959 (2009).

69. Griesbeck, M. et al. Sex differences in plasmacytoid dendritic cell levels of IRF5 drive higher IFN-alpha production in women. J. Immunol. 195, 5327-5336 (2015).

70. Laffont, S, et al. X-Chromosome complement and estrogen receptor signaling independently contribute to the enhanced TLR7-mediated IFN-alpha production of plasmacytoid dendritic cells from women. J. Immunol. 193, 5444-5452 (2014).

71. Seillet, C et al. The TLR-mediated response of plasmacytoid dendritic cells is positively regulated by estradiol in vivo through cell-intrinsic estrogen receptor alpha signaling. Blood 119, 454-464 (2012).

72. Haagmans, B. L. et al. Pegylated interferon-alpha protects type 1 pneumocytes against SARS coronavirus infection in macaques. Nat. Med. 10 290-293 (2004).

73. Blanco-Melo, D. N.-P. et al. Imbalanced host response to SARS-CoV-2 drives development of COVID-19. Cell https://doi.org/10.1016/j.cell.2020.04.026 (2020).

74. Sallard, E., Lescure, F. X., Yazdanpanah, Y., Mentre, F. $\&$ Peiffer-Smadja, N. Type 1 interferons as a potential treatment against COVID-19. Antivir. Res. 178, 104791 (2020).

75. Xu, K. et al. Factors associated with prolonged viral RNA shedding in patients with COVID-19. Clin. Infect. Dis. https://doi.org/10.1093/cid/ciaa351 (2020).

76. Zheng, S. et al. Viral load dynamics and disease severity in patients infected with SARS-CoV-2 in Zhejiang province, China, January-March 2020: retrospective cohort study. BMJ 369, m1443 (2020)

77. Sakiani, S., Olsen, N. J. \& Kovacs, W. J. Gonadal steroids and humoral immunity. Nat. Rev. Endocrinol. 9, 56-62 (2013)

78. Pauklin, S. Sernandez, I. V., Bachmann, G., Ramiro, A. R. \& Petersen-Mahrt, S. K. Estrogen directly activates AID transcription and function. J. Exp. Med. 206, 99-111 (2009).

79. Bynoe, M. S., Grimaldi, C. M. \& Diamond, B. Estrogen up-regulates $\mathrm{BCl}-2$ and blocks tolerance induction of naïve B cells. Proc. Natl Acad. Sci. USA 97, 2703-2708 (2000).

80. Grimaldi, C. M., Cleary, J., Dagtas, A. S., Moussai, D. $\&$ Diamond, B. Estrogen alters thresholds for B cell apoptosis and activation. J. Clin. Invest. 109 1625-1633 (2002).

81. Grimaldi, C. M., Jeganathan, V. \& Diamond, B. Hormonal regulation of $B$ cell development: $17 \beta$-estradiol impairs negative selection of high-affinity DNA-reactive B cells at more than one developmental checkpoint. J. Immunol. 176, 2703-2710 (2006).

82. Hill, L., Jeganathan, V., Chinnasamy, P., Grimaldi, C. $\&$ Diamond, B. Differential roles of estrogen receptors $\alpha$ and $\beta$ in control of B-cell maturation and selection. Mol. Med. 17, 211-220 (2011)

83. Zhao, R. et al. A GPR174-CCL21 module imparts sexual dimorphism to humoral immunity. Nature $\mathbf{5 7 7}$ 416-20. (2020)

84. To, K. K. et al. Temporal profiles of viral load in posterior oropharyngeal saliva samples and serum antibody responses during infection by SARS-CoV-2 an observational cohort study. Lancet Infect. Dis. 20 565-74. (2020). 
85. Wolfel, R. et al. Virological assessment of hospitalized patients with COVID-2019. Nature https://doi.org 10.1038/s41586-020-2196-x (2020).

86. Shen, C. et al. Treatment of 5 critically ill patients with COVID-19 with convalescent plasma. JAMA 323, 1582-1589 (2020).

87. Flanagan, K. L., Fink, A. L., Plebanski, M. \& Klein, S. L. Sex and gender differences in the outcomes of vaccination over the life course. Annu. Rev. Cell Dev. Biol. 33, 577-99. (2017).

88. Dragin, N. et al. Estrogen-mediated downregulation of AIRE influences sexual dimorphism in autoimmune diseases. J. Clin. Invest. 126, 1525-1537 (2016).

89. Polanczyk, M. J. et al. Cutting edge: estrogen drives expansion of the CD4+CD25+ regulatory T cell compartment. J. Immunol. 173, 2227-2230 (2004).

90. Tai, P. et al. Induction of regulatory T cells by physiological level estrogen. J. Cell Physiol. 214, 456-464 (2008)

91. Zhu, M. L. et al. Sex bias in CNS autoimmune disease mediated by androgen control of autoimmune regulator. Nat. Commun. 7, 11350 (2016).

92. Page, S. T. et al. Effect of medical castration on CD4+ CD25+ T cells, CD8+ T cell IFN-gamma expression, and NK cells: a physiological role for testosterone and/or its metabolites. Am. J. Physiol. Endocrinol. Metab. 290, E856-E863 (2006)

93. Hewagama, A., Patel, D., Yarlagadda, S. Strickland, F. M. \& Richardson, B. C. Stronger inflammatory/cytotoxic T-cell response in women identified by microarray analysis. Genes. Immun. 10 509-516 (2009).

94. Yee Mon, K. J. et al. Differential sensitivity to IL-12 drives sex-specific differences in the CD8+ T cell response to infection. Immunohorizons 3, 121-132 (2019).

95. Qu, K. et al. Individuality and variation of personal regulomes in primary human T cells. Cell Syst. 1, 51-61 (2015).

96. Wang, J. et al. Unusual maintenance of $X$ chromosome inactivation predisposes female lymphocytes for increased expression from the inactive X. Proc. Nat Acad. Sci. USA 113, E2029-E2038 (2016).

97. Janice Oh, H. L., Ken-En Gan, S., Bertoletti, A. $\&$ Tan, Y. J. Understanding the T cell immune response in SARS coronavirus infection. Emerg. Microbes Infect. 1, e23 (2012).
98. Chen, J. et al. Cellular immune responses to severe acute respiratory syndrome coronavirus (SARS-CoV) infection in senescent BALB/c mice: CD4+ T cells are important in control of SARS-CoV infection. J. Virol. 84, 1289-1301 (2010).

99. Alosaimi, B et al. MERS-CoV infection is associated with downregulation of genes encoding Th1 and Th2 cytokines/chemokines and elevated inflammatory innate immune response in the lower respiratory tract. Cytokine 126, 154895 (2020)

100. Ruan, Q. Yang, K., Wang, W., Jiang, L. \& Song, J. Clinical predictors of mortality due to COVID-19 based on an analysis of data of 150 patients from Wuhan, China. Intensive Care Med. 46, 846-848 (2020).

101. Yang, X. et al. Clinical course and outcomes of critically ill patients with SARS-CoV-2 pneumonia in Wuhan, China: a single-centered, retrospective, observational study. Lancet Respir. Med. 8, 475-481. (2020).

102. Halter, S. et al. T regulatory cells activation and distribution are modified in critically ill patients with acute respiratory distress syndrome: a prospective single-centre observational study. Anaesth. Crit. Care Pain. Med. 39, 35-44 (2020).

103. Barton, L. M., Duval, E. J., Stroberg, E., Ghosh, S. \& Mukhopadhyay, S. COVID-19 autopsies, Oklahoma, USA. Am. J. Clin. Pathol. 153, 725-33. (2020).

104. Tian, S. et al. Pulmonary pathology of early-phase 2019 novel coronavirus (COVID-19) pneumonia in two patients with lung cancer. J. Thorac. Oncol. 15 , 700-704 (2020)

105. Tian, S. et al. Pathological study of the 2019 novel coronavirus disease (COVID-19) through postmortem core biopsies. Mod. Pathol. https://doi.org/10.1038/ s41379-020-0536-x (2020)

106. Heffernan, D. S. et al. Gender and acute respiratory distress syndrome in critically injured adults: a prospective study. J. Trauma 71, 878-883 (2011).

107. Liu, T. et al. The influence of sex on outcomes in trauma patients: a meta-analysis. Am. J. Surg. 210, 911-921 (2015).

108. Falagas, M. E., Mourtzoukou, E. G. \& Vardakas, K. Z. Sex differences in the incidence and severity of respiratory tract infections. Respir. Med. 101, 1845-1863 (2007).

109. Robinson, D. P., Hall, O. J., Nilles, T. L., Bream, J. H. $\&$ Klein, S. L. 17 beta-estradiol protects females against influenza by recruiting neutrophils and increasing virus-specific CD8 T cell responses in the lungs. J. Virol. 88, 4711-4720 (2014).

110. Giamarellos-Bourboulis, E. J. et al. Complex immune dysregulation in COVID-19 patients with severe respiratory failure. Cell Host Microbe https://doi.org/ 10.1016/j.chom.2020.04.009 (2020).

111. Naugler, W. E. et al. Gender disparity in liver cancer due to sex differences in MyD88-dependent IL-6 production. Science 317, 121-124 (2007).

112. Bischof, E., Wolfe, J. \& Klein, S. L. Clinical trials for COVID-19 should include sex as a variable. J. Clin. Invest. https://doi.org/10.1172/JCI139306 (2020).

113. Canadian Institutes of Health Research. Why sex and gender need to be considered in COVID-19 research. CIHR https://cihr-irsc.gc.ca/e/51939.html (2020).

114. Klein, S. L. et al. Opinion: sex inclusion in basic research drives discovery. Proc. Natl Acad. Sci. USA 112, 5257-5258 (2015)

115. Tannenbaum, C., Ellis, R. P., Eyssel, F., Zou, J. $\&$ Schiebinger, L. Sex and gender analysis improves science and engineering. Nature 575, 137-46. (2019).

116. Bartz, D. et al. Clinical advances in sex- and gender-informed medicine to improve the health of all: a review. JAMA Intern. Med. 180, 574-583 (2020).

Author contributions

The authors contributed equally to all aspects of the article.

Competing interests

The authors declare no competing interests.

Peer review information

Nature Reviews Immunology thanks E. Fish, P. McCombe and the other, anonymous, reviewer(s) for their contribution to the peer review of this work.

Publisher's note

Springer Nature remains neutral with regard to jurisdictional claims in published maps and institutional affiliations.

RELATED LINKS

Global Health 50/50: http://globalhealth5050.org/covid19/

(c) Springer Nature Limited 2020 